\title{
Determination of the Bending Rigidity of Graphene via Electrostatic Actuation of Buckled Membranes
}

\author{
Niklas Lindahl, ${ }^{\dagger, \#}$ Daniel Midtvedt, ${ }^{\ddagger}, \#$ Johannes Svensson, ${ }^{\S}$ Oleg A. Nerushev, ${ }^{\|}$Niclas Lindvall, ${ }^{\perp}$ \\ Andreas Isacsson, ${ }^{\ddagger}$ and Eleanor E. B. Campbell ${ }^{*}, \|, \Phi$ \\ ${ }^{\dagger}$ Department of Physics, University of Gothenburg, SE-41296 Göteborg, Sweden \\ ${ }^{\ddagger}$ Department of Applied Physics, Chalmers University of Technology, SE-41296 Göteborg, Sweden \\ ${ }^{\S}$ Electrical and Information Technology, Lund University, SE-22 100 Lund, Sweden \\ "EaStCHEM, School of Chemistry, Edinburgh University, Edinburgh EH9 3JJ, Scotland \\ ${ }^{\perp}$ Department of Microtechnology and Nanoscience, Chalmers University of Technology, SE-41296 Göteborg, Sweden

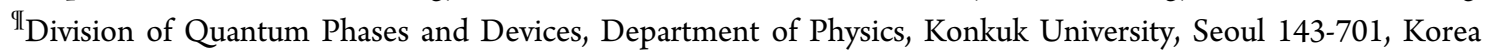

Supporting Information
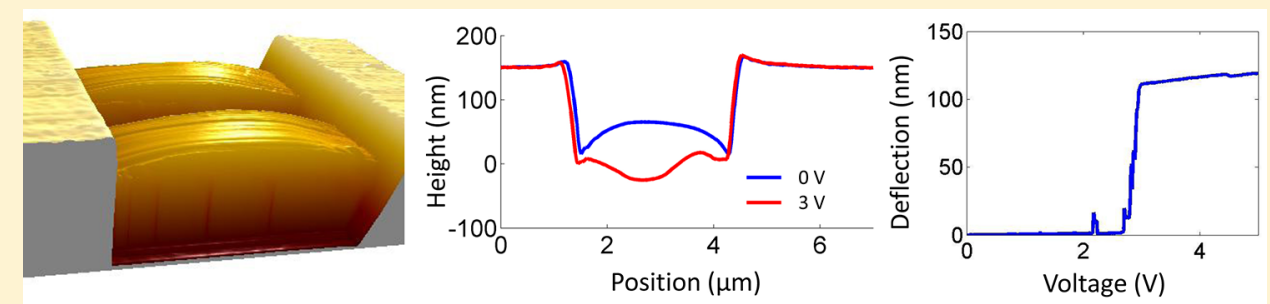

ABSTRACT: Classical continuum mechanics is used extensively to predict the properties of nanoscale materials such as graphene. The bending rigidity, $\kappa$, is an important parameter that is used, for example, to predict the performance of graphene nanoelectromechanical devices and also ripple formation. Despite its importance, there is a large spread in the theoretical predictions of $\kappa$ for few-layer graphene. We have used the snap-through behavior of convex buckled graphene membranes under the application of electrostatic pressure to determine experimentally values of $\kappa$ for double-layer graphene membranes. We demonstrate how to prepare convex-buckled suspended graphene ribbons and fully clamped suspended membranes and show how the determination of the curvature of the membranes and the critical snap-through voltage, using AFM, allows us to extract $\kappa$. The bending rigidity of bilayer graphene membranes under ambient conditions was determined to be $35.5_{-15.0}^{+20.0} \mathrm{eV}$. Monolayers are shown to have significantly lower $\kappa$ than bilayers.

KEYWORDS: Few-layer graphene, bending rigidity, buckled membranes

$\mathrm{T}$ he small mass and atomic-scale thickness of graphene membranes make them highly suitable for nanoelectromechanical devices such as, for example, mass sensors, highfrequency resonators or memory elements. Although only atomically thick, many of the mechanical properties of graphene membranes can be described by classical continuum mechanics. ${ }^{1,2}$ An important parameter for predicting the performance and linearity of graphene nanoelectromechanical devices $^{3}$ as well as for describing ripple formation ${ }^{4}$ and other properties such as electron scattering mechanisms, ${ }^{5}$ is the bending rigidity, $\kappa$. Despite the importance of this parameter it has so far only been estimated indirectly for monolayer graphene from the phonon spectrum of graphite, ${ }^{6}$ estimated from AFM measurements for multilayer graphene (>8 layers), ${ }^{7,8}$ or predicted from $a b$ initio calculations ${ }^{1,9}$ or bondorder potential models. ${ }^{3,10}$ Here, we employ a new approach to the experimental determination of $\kappa$ by exploiting the snapthrough instability in prebuckled graphene membranes. We demonstrate the reproducible fabrication of convex buckled graphene membranes by controlling the thermal stress during the fabrication procedure and show the abrupt switching from convex to concave geometry that occurs when electrostatic pressure is applied via an underlying gate electrode and how this can be used to extract $\kappa$. The value that we obtain for bilayer graphene, $\kappa=35.5_{-15.0}^{+20.0} \mathrm{eV}$, lies between the two extreme theoretical predictions of $\kappa=160 \mathrm{eV}^{9}$ (from zero-temperature $a b$ initio calculations) and $\kappa=3 \mathrm{eV}$ (assuming independent monolayers at room temperature). ${ }^{11}$

For deformations on a scale large compared to the inter atomic spacing, the mechanical properties of single-layer graphene (SLG) as well as few-layer graphene (FLG) can be modeled using the theory of two-dimensional (2D) membranes. In this theory, the effective free energy is a functional of

Received: March 20, 2012

Revised: May 23, 2012

Published: June 18, 2012 
the transverse displacement $w$ and the in-plane displacement vector $u^{12}$

$$
F=\frac{1}{2} \int \mathrm{d}^{2} x\left[\kappa\left(\nabla^{2} w\right)^{2}+\mu u_{\alpha \beta}^{2}+\frac{\lambda}{2} u_{\alpha \alpha}^{2}\right]
$$

Here $u_{\alpha \beta}=\left(\partial_{\alpha} u_{\beta}+\partial_{\beta} u_{\alpha}+\partial_{\alpha} w \partial_{\beta} w\right) / 2$ is the strain tensor and the indices $\alpha$ and $\beta$ run over the Cartesian coordinates $x$ and $y$ in the plane of the graphene sheet. Repeated indices are summed over. The material parameters in (1) are the bending rigidity $\kappa$ and the Lamé coefficients $\mu$ and $\lambda$. Because of thermal fluctuations, for instance ripples, these parameters will in general depend on temperature $T$. While the combination $C \approx$ $(\lambda+2 \mu)$ corresponding to the $2 \mathrm{D}$ elastic modulus has been measured at room temperature to be close to its predicted zerotemperature value for graphene $C \approx 340 \mathrm{~N} \mathrm{~m}^{-11,13}$ a direct measurement of the bending rigidity $\kappa$ is lacking both for SLG as well as FLG. The value often quoted for the bending rigidity of monolayer graphene $(\kappa=1.2 \mathrm{eV})$ was estimated from the phonon spectrum of graphite. ${ }^{6}$

Using eq 1 is equivalent to treating the suspended membrane as a thin plate with a Young's modulus E, Poisson's ratio $\nu$, and thickness $h$, if we make the identifications

$$
\frac{E h}{1-\nu^{2}}=\lambda+2 \mu, \nu=\frac{\lambda}{\lambda+2 \mu}, \quad \kappa=\frac{E h^{3}}{12\left(1-\nu^{2}\right)}
$$

The parameters $E h, \nu$, and $h$ are then uniquely mapped onto the parameters $\kappa, \mu$, and $\lambda$ of eq 1 . Here, $E$ is not independent of $h$, rather it is the product $E h$, which is determined. Often, as in experiments on SLG and FLG nano resonators, ${ }^{14-16}$ in-plane stress dominates and the first term in (1) can be disregarded. In such cases one often sets $h=3.4 \AA$ for SLG, the interplanar distance between the atomic layers in graphite. As $E h \approx 340 \mathrm{~N}$ $\mathrm{m}^{-1}, 1,13$ this leads to $E \approx 1 \mathrm{TPa}$. However, from eqs 2 these values of $E$ and $h$, together with $\nu \approx 0.16,{ }^{17}$ give $\kappa \approx 20 \mathrm{eV}$, an order of magnitude larger than the $\sim 1 \mathrm{eV}$ estimated from phonon measurements and ab-initio calculations.

For SLG, the discrepancy stems from the different physical origins of bending rigidity in SLG and continuum thin plates. In thin plates, the nonzero $\kappa$ originates from the compression/ extension of the medium on either side of the neutral surface. In SLG, there is not a continuum in the direction perpendicular to the membrane and bond-order models have indicated two physical origins. One is due to the bond angle effect and the other results from the bond-order term associated with the dihedral angles. ${ }^{10}$ Indeed, bond-order calculations give for SLG a $T=0$ value $\kappa \approx 1.4 \mathrm{eV}$ that is close to $a b$ initio predictions of $1.46^{1}$ or $1.6 \mathrm{eV}^{9}$ and to the experimental value derived from the phonon spectrum of graphite $(1.2 \mathrm{eV}) .{ }^{6}$ For $T>0$, ripples in SLG are predicted to increase $\kappa$ at long wavelengths. ${ }^{4}$ For FLG, one expects to approach the thin plate theory scaling, $\kappa \propto h^{3}$, as the number of layers grows. For bilayer graphene (BLG) and trilayer graphene (TLG), ab initio calculations and estimates using bond-order potentials have, for $T=0 \mathrm{~K}$, predicted $\kappa_{\mathrm{BLG}} \approx$ $160-180 \mathrm{eV}$ and $\kappa_{\mathrm{TLG}} \approx 660-690 \mathrm{eV} .^{9,11}$ In these calculations, the contributions to $\kappa$ come mainly from the energy required to stretch/compress the upper/lower graphene layer as in thin plate theory. However, in contrast to SLG, where thermal fluctuations are predicted to increase $\kappa$, for FLG at $T>0 \mathrm{~K}$, local thermal interplane distance fluctuations have been predicted to soften the bending rigidity, ${ }^{12}$ approaching $\kappa_{\mathrm{BLG}}$ $\approx 2 \kappa_{\mathrm{SLG}} \approx 3 \mathrm{eV}$ at room temperature. The large deviation between $\kappa(T=0 \mathrm{~K}) \sim 10^{2} \mathrm{eV}$ and the finite temperature estimate of a few electronvolts makes it important to experimentally determine the value of $\kappa$ for $T>0 \mathrm{~K}$.

Nanoindentation measurements have been used to extract values for the bending rigidities of suspended multilayer graphene ( $\geq 8$ layers). ${ }^{7}$ In such experiments, a force versus deflection curve is obtained by pushing the suspended part of the sample with an AFM-tip. However, extracting $\kappa$ in this way is problematic for two reasons. The first comes from the large in-plane stiffness of graphene that implies that a deformation of $w \sim 1 \AA$ will cause stretching contributions to dominate. Second, suspended samples are commonly under finite tensile strain due to electrode adhesion effects. Despite the inherent difficulties with the technique, the extracted values fit reasonably well to the results of modeling the suspended membranes as thin plates. Using eq 2 to fit the data of Poot and van der Zant ${ }^{7}$ yields (measuring $h$ in $\mathrm{nm}$ ) $\kappa=570 h^{3} \mathrm{eV}, E=$ $0.92 \mathrm{TPa}$ and $\nu=0.16$. A second AFM technique studies the deformation that graphene layers produce on a microcorrugated elastic surface. ${ }^{8}$ A model is then used to extract a so-called flattening factor that can be related to the bending rigidity as a function of the number of layers. This technique also contains uncertainty with respect to the influence of tension and interface strength. The best fit for the dependence of $\kappa$ on $h$, yields $\kappa=182 h^{3} \mathrm{eV}$ (with $h$ in $\mathrm{nm}$ ).

In this Letter, we exploit snap-through instabilities in prebuckled graphene. In the fabrication of suspended samples (beams and circular/elliptic drums), a controlled compressive strain is built in before under-etching the devices to produce the suspended SLG and FLG. When released, this leads to convex buckled geometries with zero built-in strain. In most of our samples, the suspended regions are buckled upward away from the substrate. We attribute this to adhesive forces between the graphene and the electrodes. This effect of adhesion to the clamping points, which in our case is a result of under etching, has been observed previously for graphene on top of holes. ${ }^{13}$ By biasing the backgate, an electrostatic pressure is applied to the membranes. Our method is based on relating the snapthrough voltage to the local curvature, measured by AFM, and observing at what pressure the membrane undergoes a buckling deformation.

To extract $\kappa$ we note, from the analogy with thin plate theory, that our buckled membranes are expected to show similar deformation properties to those of convex shells. Fully clamped shells display buckling instability under external pressure that is observed as a snap-through from locally convex to locally concave buckling ${ }^{18}$ at a critical pressure $p_{c}$. The development of a shell under external pressure is sketched in Figure 1a-c. When pressure is applied, a shell with nonzero Gaussian curvature deforms first locally in the region around the structurally weakest point (see Figure 1a). While this deformation lowers the energy due to pressure-volume (PV) work, it is at the expense of increasing the contributions from elastic energy (mainly stretching/compression). For small deformations, the balance between the elastic energy and the PV-term makes the system stable. For a deformed region larger than a critical size however, it becomes energetically favorable to form a large angle bend (Figure $1 \mathrm{~b}$ ) surrounding an inward bulge. Following Pogorelov ${ }^{18}$ we assume that this inward bulge forms a mirror reflection of the original surface in a plane perpendicular to the symmetry axis. Inside the bulge, the curvature of the deformed shell is then identical in magnitude to the initial surface. Hence, in this region the elastic energy density remains unchanged. The major contribution to it comes 


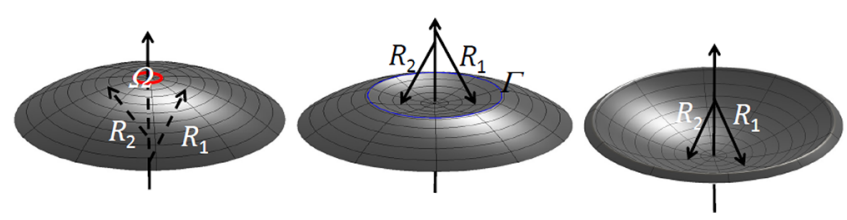

Figure 1. Schematic pictures showing the snap-through of a convex shell. (Left) For pressure smaller than a critical pressure $p_{\mathcal{c}}$ a small finite deformation is formed in the region $\Omega$. (Middle) As the critical pressure $p_{c}$ is reached it becomes energetically favorable to form a concave region where the elastic energy is confined to a narrow region around the annulus $\Gamma$. (Right) As the concave configuration in middle panel is unstable, the deformation propagates outward, the membrane "snaps-through". By measuring the radii of curvature $R_{1,2}$ and relating the pressure to the applied backgate voltage when the membrane snaps though, $p_{\mathrm{c}}$ can be determined.

instead from a narrow region around the edge of the bulge, in the figure denoted by $\Gamma$. This energy is given by $U=(4 \kappa n(\lambda+$ $2 \mu))^{1 / 2} \int \mathrm{d} s k_{\mathrm{n}}^{2} / k_{\Gamma}$, where $k_{\Gamma}$ is the curvature of $\Gamma, n$ is the number of graphene layers, and $k_{\mathrm{n}}$ is the normal curvature of the shell along $\Gamma$. As the work done by the pressure is proportional to the area inside the bulge, this state is unstable and the edge of the bulge propagates outward. This continues until the propagation is hindered by the edges or defects in the sample at which point the shell is said to have "snapped through" (Figure 1c). A detailed calculation, following Pogorelov, ${ }^{18}$ for our fully clamped structures gives the following expression for the pressure at which the critical deformation is reached

$$
p_{c}=\frac{4 \sqrt{\kappa n(\lambda+2 \mu)}}{R_{1} R_{2}}
$$

Here $R_{1}$ and $R_{2}$ are the principal radii of curvature (in orthogonal directions) at the point where the instability starts. As $(\lambda+2 \mu) \approx 340 \mathrm{Nm}^{-1}$ we can use relation 3 to extract $\kappa$ from measured values of $p_{\mathrm{c}}$ and $R_{1,2}$. For the beams, the inclusion of free edges makes the problem intractable analytically. However, following the argumentation outlined in Landau and Lifschitz, ${ }^{19}$ the scaling of the critical pressure can still be obtained. We find that the scaling of the critical pressure for the beams is

$$
p_{c} \propto \frac{\sqrt{\kappa n(\lambda+2 \mu)}}{\sqrt{R_{1}^{3} R_{2}}}
$$

where $R_{1}$ is the curvature in the direction along the long axis of the beam and $R_{2}$ is the curvature in the perpendicular direction.

In order to prepare convex membranes, graphene was obtained from mechanical exfoliation on silicon substrates with $295 \mathrm{~nm}$ oxide. ${ }^{20}$ Optical microscopy was used for finding the location of flakes with a suitable shape and number of layers. The number of layers was determined by the optical contrast and confirmed by Raman spectroscopy on selected samples. Graphene flakes were shaped into the desired geometry using electron-beam lithography (EBL) to pattern a resist mask (positive resist PMMA). The resist was typically baked at 160 ${ }^{\circ} \mathrm{C}$ to remove solvents after spin-coating. A low-power oxygenplasma that removed $10 \mathrm{~nm}$ of resist was used to etch the nonmasked graphene. The resist mask was removed in acetone leaving the patterned graphene. A bilayer resist composed of bottom-layer copolymer MMA-MAA and top-layer PMMA was used to pattern the electrodes used to clamp and electrically contact the graphene structures. Evaporation of $3 \mathrm{~nm} \mathrm{Cr}$ and $150 \mathrm{~nm} \mathrm{Au}$ was done using e-gun evaporation. $\mathrm{Cr}$ was used as adhesion layer since it is compatible with HF-etching. A relatively thick layer of $\mathrm{Au}$ was used to avoid electrostatic actuation of the suspended part of the electrodes. Bilayer resist was used to ensure an under-cut, facilitating lift-off after evaporation. Lift-off was done using ultrasonic agitation in hot acetone. To suspend the graphene beams, the substrate was wet-etched using HF. During etching the electrodes act as an etch-mask. The etchant penetrates freely under the graphene beam. Conditions were chosen to etch away $225 \mathrm{~nm}$ of the underlying oxide under the entire patterned graphene structure, including the graphene covered by the electrodes. Thus to avoid excessive under-etching of electrodes, causing their electrostatic actuation during the later experiments, graphene patterns were formed first, making it possible to control the overlap distance between the electrodes and the graphene. Rinsing was done in milli- $Q$ followed by IPA. After etching, critical point drying was used to avoid collapse of the membranes due to surface tension effects during drying. Care was taken to ensure that there were no detectable resist residues remaining on the graphene that may influence the bending rigidity measurements. It was possible to observe resist residue on supported graphene prior to substrate etching. This showed up as bright spots in the AFM height image and as dark spots in the AFM phase image. However, after etching in HF, this structure was usually removed. In order to check that any remaining resist residue did not influence the results of the bending rigidity measurements, we also annealed some samples in $\mathrm{Ar} / \mathrm{H}_{2}$ and confirmed that there was no significant difference in the determined bending rigidity.

Raman spectra were obtained using a Renishaw microRaman spectrometer with a $514 \mathrm{~nm}$ excitation laser and spectral resolution better than $1 \mathrm{~cm}^{-1}$. The shape of the $2 \mathrm{D}$ peak was used to confirm the number of graphene layers, estimated from the optical contrast. Raman spectra were also measured in situ on the same graphene flake during heating from room temperature to $200{ }^{\circ} \mathrm{C}$ and during cooling back to room temperature to determine the extent of thermal stress. The results are shown in the Supporting Information.

Electrostatic actuation of the suspended graphene was achieved by applying a voltage, $V_{\mathrm{bg}}$, to the silicon back-gate while keeping the graphene grounded. The depth of etching was chosen to leave some remaining insulating $\mathrm{SiO}_{2}(70 \mathrm{~nm})$ to avoid a short-circuit between the graphene and the back electrode. Similar to previous studies of multiwalled carbon nanotubes $^{21}$ and multilayered graphene, ${ }^{22}$ electrostatic deflection was imaged in situ using AFM. The AFM was used in noncontact mode and measurements were carried out in air at $22{ }^{\circ} \mathrm{C}$. To reduce the interaction between the suspended graphene and the AFM cantilever both were grounded. The AFM is operated under conditions where the force of interaction with the substrate is low and also operates at a frequency approximately 2 orders of magnitude lower than the resonant frequency of the membranes. We can therefore discount the influence of tip interactions for the substrates discussed in this paper.

Figure 2 shows two suspended BLG beams fabricated using the techniques detailed above. It is easier to visualize the convex buckling with beam geometries rather than the fully clamped structures that are needed to compare quantitatively with the model discussed above. Figure $2 \mathrm{a}$ shows an exaggerated schematic illustrating the way in which the beams are attached 

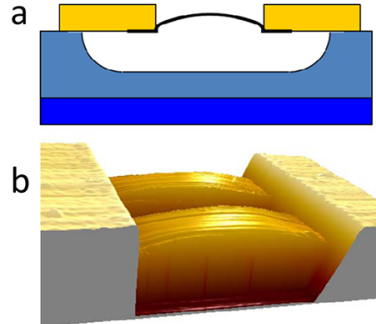

C

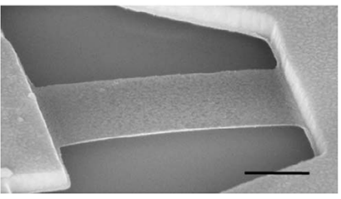

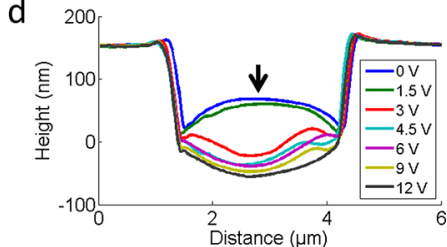

e

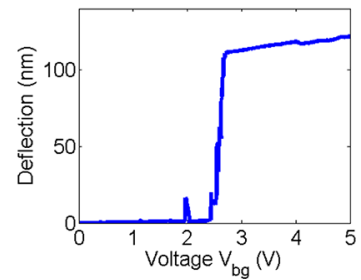

Figure 2. (a) Schematic picture of under-etched suspended graphene beams. When the graphene is under-etched it is released. The built-in compressive strain together with the adhesion to the electrodes will result in a buckled shape with the graphene curving away from the substrate. (b) AFM scan of two suspended bilayer beams showing convex buckled shapes. The length of the beams is $2 \mu \mathrm{m}$ and the center of the beam is ca. $25 \mathrm{~nm}$ higher than the clamped edges. (c) SEM image of a suspended beam with visible upward buckling. The scale bar is $1 \mu \mathrm{m}$. (d) Line scans along the center of one of the beams as a function of $V_{\mathrm{bg}}$. (e) Deflection versus $V_{\mathrm{bg}}$ plot for the same beam measured at the center of the beam, position indicated by the arrow in (d). The deflection is defined as the deviation from the equilibrium distance at $V_{\mathrm{bg}}=0 \mathrm{~V}$.

to the electrodes and the convex curvature produced when the substrate is etched away from the graphene. From the measured AFM profile in Figure $2 b$ it can be clearly seen that the beams are buckled to give a convex geometry. In this example, the lengths of the beams are on average $0.12 \%$ longer than the horizontal end-to-end-distance.

The buckling is just detectable in the SEM picture in Figure $2 \mathrm{c}$ where it is also possible to observe the under-etching of the electrodes. The abrupt snap-through predicted by the model for the fully clamped geometry can also be seen with the beams. Figure $2 \mathrm{~d}$ shows line scans along one of the beams as a function of increasing voltage applied to a back gate and thus increasing electrostatic pressure applied to the suspended beam. The abruptness of the transition is clearly seen in Figure 2e where the height of the central position of the beam is plotted as a function of the back gate voltage. The deflection curve shown in Figure $2 \mathrm{e}$ is obtained by placing the AFM tip at a fixed position in the center of the beam and sweeping $V_{\mathrm{bg}}$ while measuring the deflection from the initial position. This shows that there is a sharp snap-through from convex to concave buckling where the beam deflects a large distance for a small change in $V_{\mathrm{bg}}$. This is quite different behavior to that observed for suspended graphene beams that do not have this initial buckled shape. As we have shown previously, in that case there is a continuous deflection of the suspended membrane until it snaps to contact with the underlying substrate when the pull-in voltage has been exceeded. ${ }^{23}$

The observed buckling is a consequence of the mismatch between the thermal expansion coefficients of the graphene and a

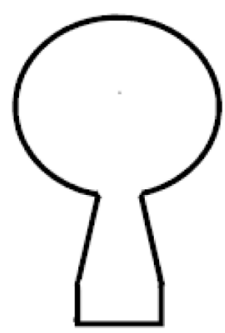

$\mathrm{b}$

d

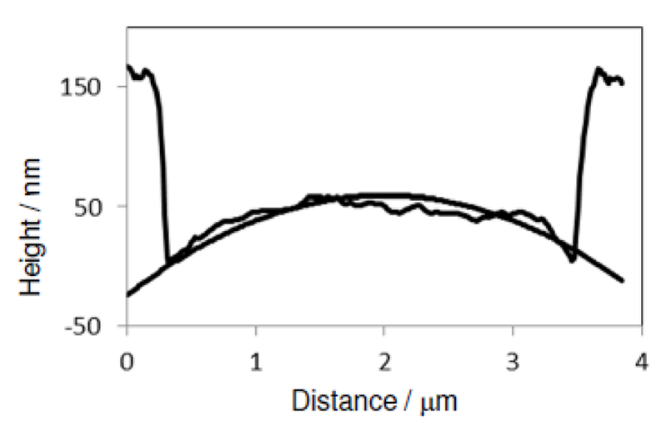

$\mathrm{C}$

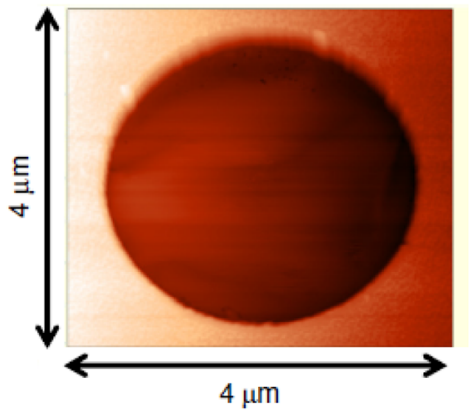

e

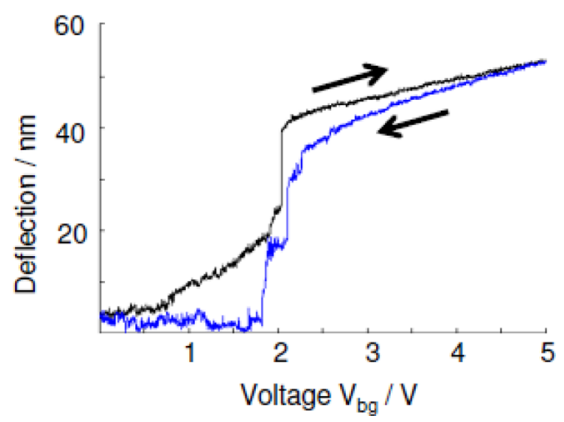

Figure 3. (a) Graphene "frying pan" pattern used to fabricate fully clamped circular membranes. (b) A square electrode with a hole in the middle is patterned on top of the graphene, light-gray in figure. The graphene is clamped by the electrode in the midgray areas and left exposed in dark-gray areas. When the substrate is etched, the bottom-side of the handle of the "frying pan" is exposed outside the electrode. The etchant is able to penetrate freely under the graphene all the way underneath the shape of the "frying pan" and continues to under-etch the electrode, thus suspending the whole area inside the dashed line in Figure 5b. (c) AFM scan of a suspended circular membrane. (d) Line scan across the center of the suspended membrane corresponding to the dashed line in (b). The smooth line illustrates the fitted curvature. (e) Deflection versus $V_{\mathrm{bg}}$ curve obtained with the AFM tip at the center of the suspended fully clamped BLG membrane. The deflection is defined as the deviation from the equilibrium distance at $V_{\mathrm{bg}}=0 \mathrm{~V}$ measured at the center of the beam. 
the underlying $\mathrm{SiO}_{2}$ substrate due to thermal cycling prior to etching (see above). Hence, before under-etching the thermal cycling results in a compressive strain in the graphene lying on the $\mathrm{SiO}_{2}$. The buckling arises upon release (etching) as the built-in compressive strain causes the suspended sheet to be slightly larger than the exposed hole. Evidence for this is provided in the form of temperature-dependent Raman measurements detailed in the Supporting Information. The results are very reproducible in the sense that suspended membranes made from the same graphene sheet and having undergone the same thermal cycling show the same amount of built-in compressive strain before under etching and the same relative extension after under-etching. Hence, the buckling in our samples could be controlled by the extent of thermal cycling to which the substrate was exposed. In particular, for samples where thermal cycling was avoided during lithography no buckling was observed (see Supporting Information).

The beam structures clearly show a curvature along the long axis of the beam (measured between the two clamping electrodes) as is most apparent in Figure $2 c$ and the rapid snap-through predicted for convex shells. However, since the beams are not fully clamped around their circumference, it is not possible to apply the model as developed by Pogorelov to extract an absolute value of $\kappa$. For this reason, we have also fabricated fully clamped membranes of circular or elliptical shape. In this case, the membranes clearly show radii of curvature in two orthogonal directions and it is possible to treat them as deforming convex shells using eq 3. An example of a circular suspended BLG membrane is shown in Figure 3.

The electrostatic pressure applied in the experiments can be calculated from the parallel plate model. Then, from eq 3 the bending rigidity $\kappa$ is given by

$$
\kappa=\left(\frac{R_{1} R_{2}}{d^{2}}\right)^{2} \frac{\varepsilon_{0}^{2} V_{c}^{4}}{64 n(\lambda+2 \mu)}
$$

where $d$ is the effective distance to the gate $(243 \mathrm{~nm}$, accounting for the dielectric constant of the remaining oxide layer), $\varepsilon_{0}$ is the vacuum permittivity, and $V_{c}$ is the critical voltage at which snap-through occurs. The validity of the parallel plate model has been checked with FEM simulations (Supporting Information). Using $(\lambda+2 \mu)=340 \mathrm{Nm}^{-1}$ we plot in Figure $4 \log \left[\left(\varepsilon_{0} V_{c}^{4}\right) / 64 n(\lambda+2 \mu)\right]$ against $\log \left[d^{2} / R_{1} R_{2}\right]^{2}$ for the experimental devices. According to the model, the points

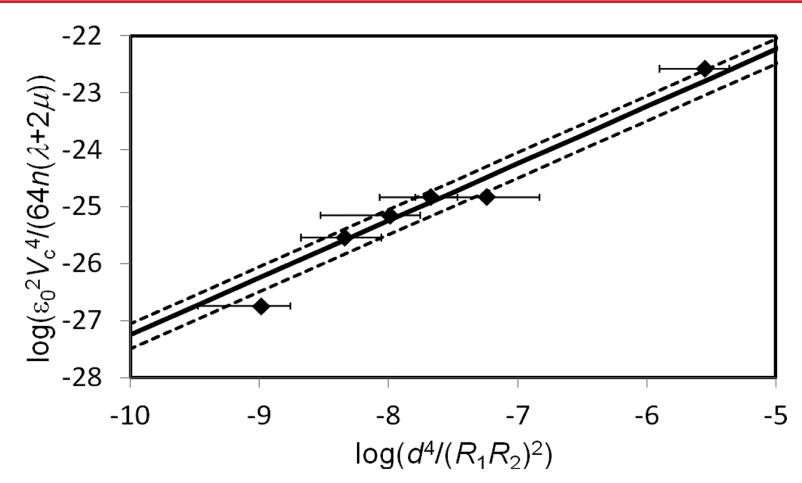

Figure 4. Plot used to determine $\kappa$ for fully clamped BLG. $\kappa$ is determined from the value of the $y$-intercept at $x=0$. Diamonds: average experimental values obtained from fitting the radii of curvature from at least six AFM line scans on each substrate, error bars indicate the standard deviation of the fitted radii. The full line is a straight-line fit to the data and the dashed lines indicate the stated error limits. should then fall along a straight line with unit slope. The bending rigidity can then be determined from the value of the $y$-axis intercept.

The results in Figure 4 are for fully clamped circular and elliptical BLG membranes. We attempted to produce similar structures with SLG membranes but this proved to be very difficult and the membranes typically broke or did not show a well-defined curvature making the analysis extremely unreliable. The principal curvatures of the BLG membranes were determined by fitting the deflection data from the AFM measurements in orthogonal directions, similar to the example shown in Figure 3d. The results are tabulated in the Supporting Information along with the values determined for the snapthrough voltage, $V_{c}$. The stated radii are the average values obtained from fitting at least six AFM line scans for each membrane with the error bars given by the standard deviation of the fitted radii. In order to extract $\kappa$, the gradient was constrained to be 1 (as expected from eq 4 and consistent with a fitted value of $1.1 \pm 0.16)$ and the intercept was determined from a least-squares fit. The fit line is shown as a full line in Figure 4 with the estimated error limits indicated by dashed lines. The value obtained for the bending rigidity is $\kappa=35.5_{-15.0}^{+20.0}$ $\mathrm{eV}$. This value is significantly lower than the value estimated from eq 2, using $E=0.92 \mathrm{TPa}$ and $h=6.8 \AA$ (giving $\kappa_{\mathrm{BLG}}=155$ $\mathrm{eV}$ ), and the values obtained from zero-temperature $a b$ initio calculations for BLG $\left(\kappa_{\mathrm{BLG}}=160-180 \mathrm{eV}^{9,11}\right)$. It is, however, considerably higher than the predicted value that assumes two independent monolayers at room temperature $\left(\kappa_{\mathrm{BLG}}=3 \mathrm{eV}\right){ }^{1{ }^{11}}$ The agreement between continuum theory and the experimental results presented here (eq 5) provides convincing evidence that the continuum theory approach (eq 1 ) is valid for BLG membranes under ambient conditions provided that one adopts a value of $\kappa$ that falls between the two extremes of the theoretical predictions.

We have also analyzed the radii of curvature (along the longaxis of the beam) and critical switching voltages for a number of doubly clamped graphene beams including SLG, BLG, and TLG (data included in Supporting Information). For the beams, the expression analogous to eq 5 is

$$
\kappa \propto \frac{R_{1}^{3} R_{2}}{d^{4}} \frac{\varepsilon_{0}^{2} V_{c}^{4}}{n(\lambda+2 \mu)}
$$

The beam data has been plotted in Figure 5 in a plot of $\log \left(V_{c}^{4}\right)$ versus $\log \left(R_{1}^{-3}\right)$, following eq 6 . The data from the BLG doubly clamped beams fall on a straight line in this plot. Assuming that the bending rigidity for the beams is identical to that for the BLG fully clamped membranes, we can estimate the value of $\kappa$ for the monolayer beams by comparing the values of the $y$-intercept on this plot. The comparison yields an estimate of $\kappa_{\mathrm{SLG}}=7.1_{-3.0}^{+4.0} \mathrm{eV}$ for the monolayer and $\kappa_{\mathrm{TLG}}=126_{-53}^{+71} \mathrm{eV}$ for the trilayer; however, it should be stressed that this is a very rough estimate due to the limited number of data points used to extract the values.

By studying the voltage-induced snap-through of convex buckled membranes and beams of suspended graphene, we have shown that the mechanical behavior of BLG membranes can be described within continuum theory by treating them as convex shells but we also show that care must be taken in the choice of the parameters to be used and it is not always appropriate to scale-down from the bulk values. The value that we obtain for the bending rigidity of BLG at room temperature under ambient conditions $\left(35.5_{-15.0}^{+20.0} \mathrm{eV}\right)$ is the first 


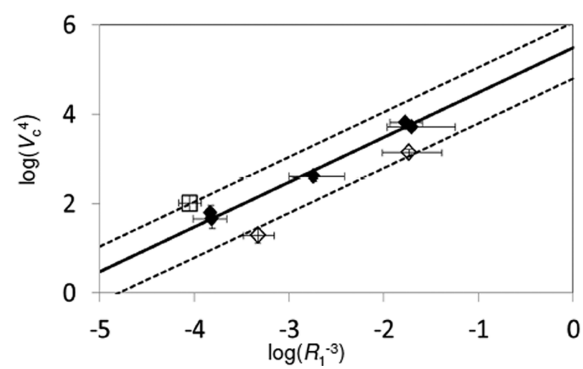

Figure 5. Data for doubly clamped beams showing the expected linear behavior for a plot of $\log \left(V_{\mathrm{c}}\right)$ versus $\log \left(R_{1}{ }^{-3}\right)$, see eqs 4 and 6. Filled diamonds, doubly clamped BLG; open diamonds, SLG; open square, TLG. The full line is a least-squares fit to the BLG data yielding an intercept of 5.5. The dashed lines show the estimated fits for the SLG (intercept 4.8) and TLG (intercept 6.05) data. The bending rigidity can be extracted by assuming that the bending rigidity of the doubly clamped BLG ribbons is identical to that of the fully clamped membranes. This yields estimated values of $\kappa_{\mathrm{SLG}}=7.1_{-3.0}^{+4.0} \mathrm{eV}$ and $\kappa_{\mathrm{TLG}}$ $=126_{-53}^{+71} \mathrm{eV}$.

experimental determination of this parameter for BLG. The value lies in between the two extreme theoretical predictions for two completely independent monolayers at finite temperature and for bilayers at $0 \mathrm{~K}$. An accurate experimental determination of $\kappa$ is crucial for understanding and correctly modeling the mechanical behavior of this important new material. The method that we present here is straightforward and can easily be extended to thicker graphene layers or other thin layer materials that can be fabricated to give similar geometries.

\section{ASSOCIATED CONTENT}

\section{S Supporting Information}

Raman studies of thermal cycling induced stress, residue test studies, tabulated radii of curvature, and critical switching voltages. This material is available free of charge via the Internet at http://pubs.acs.org.

\section{AUTHOR INFORMATION}

\section{Corresponding Author}

*E-mail: Eleanor.Campbell@ed.ac.uk.

\section{Author Contributions}

\#These authors contributed equally to the work.

\section{Notes}

The authors declare no competing financial interest.

\section{ACKNOWLEDGMENTS}

We thank Jari Kinaret for useful technical discussions. Financial support from the Swedish Strategic Research Foundation (SSF) through "NEM arrayer för elektronik- och fotonikkomponenter" (RE07-0004) is gratefully acknowledged. A.I. and D.M. acknowledge funding from the EU 7th Framework Program (FP7/2007-2013) under Grant 246026, RODIN. E.E.B.C. acknowledges financial support from the WCU program of the MEST (R31-2008-000-10057-0).

\section{REFERENCES}

(1) Kudin, K. N.; Scuseria, G. E.; Yakobson, B. I.: $\mathrm{C}_{2} \mathrm{~F}, \mathrm{BN}$ and C nanoshell elasticity from $a b$ initio computations. Phys. Rev. B 2001, 64.

(2) Cadelano, E.; Palla, P. L.; Giordano, S.; Colombo, L. Nonlinear Elasticity of Monolayer Graphene. Phys. Rev. Lett. 2009, 102, 235502.
(3) Atalaya, J.; Isacsson, A.; Kinaret, J. M. Continuum Elastic Modelling of Graphene Resonators. Nano Lett. 2008, 8, 5.

(4) Fasolino, A.; Los, J. H.; Katsnelson, M. I. Intrinsic ripples in graphene. Nat. Mater. 2007, 6, 858-861.

(5) Castro, E. V.; Ochoa, H.; Katsnelson, M. I.; Gorbachev, R. V.; Elias, D. C.; Novoselov, K. S.; Geim, A. K.; Guinea, F. Limits on Charge Carrier Mobility in Suspended Graphene due to Flexural Phonons. Phys. Rev. Lett. 2010, 105, 266601.

(6) Nicklow, R.; Wakabayashi, N.; Smith, H. G. Lattice Dynamics of Pyrolytic Graphite. Phys. Rev. B 1972, 5, 12.

(7) Poot, M.; van der Zant, H. S. J. Nanomechanical properties of few-layer graphene membranes. Appl. Phys. Lett. 2008, 92, 063111.

(8) Scharfenberg, S.; Rocklin, D. Z.; Chialvo, C.; Weaver, R. L.; Goldbart, P. M.; Mason, N. Probing the mechanical properties of graphene using a corrugated elastic substrate. Appl. Phys. Lett. 2011, 98, 091908.

(9) Zhang, D. B.; Akatyeva, E.; Dumitrica, T. Bending ultrathin graphene at the margins of continuum mechanics. Phys. Rev. Lett. 2011, 106, 255503.

(10) Lu, Q.; Arroyo, M.; Huang, R. Elastic bending modulus of monolayer graphene. J. Phys. D: Appl. Phys. 2009, 42, 102002.

(11) Koskinen, P.; Kit, O. O. Approximate modeling of spherical membranes. Phys. Rev. B 2010, 82, 235420.

(12) Zakharchenko, K. V.; Los, J. H.; Katsnelson, M. I.; Fasolino, A. Atomistic simulations of structural and thermodynamic properties of bilayer graphene. Phys. Rev. B 2010, 81, 235439.

(13) Lee, C.; Wei, X.; Kysar, J. W.; Hone, J. Measurement of the Elastic Properties and Intrinsic Strength of Monolayer Graphene. Science 2008, 321, 385-388.

(14) Garcia-Sanchez, D.; van der Zande, A. M.; Paulo, A. S.; Lassagne, B.; McEuen, P. L.; Bachtold, A. Imaging Mechanical Vibrations in Suspended Graphene Sheets. Nano Lett. 2008, 8, 13991403.

(15) Bunch, J. S.; van der Zande, A. M.; Verbridge, S. S.; Frank, I. W.; Tanenbaum, D. M.; Parpia, J. M.; Craighead, H. G.; McEuen, P. L. Electromechanical Resonators from Graphene Sheets. Science 2007, 315, 490-493.

(16) Bunch, J. S.; Verbridge, S. S.; Alden, J. S.; van der Zande, A. M.; Parpia, J. M.; Craighead, H. G.; McEuen, P. L. Impermeable Atomic Membranes from Graphene Sheets. Nano Lett. 2008, 8, 2458-2462.

(17) Blakslee, O. L.; Proctor, D. G.; Seldin, E. J.; Spence, G. B.; Weng, T. Elastic constants of compression-annealed pyrolytic graphite. J. Appl. Phys. 1970, 41, 3373-3382.

(18) Pogorelov, A. V. Bending of Surfaces and Stability of Shells, 1st ed.; Amercan Mathematical Society: Providence, RI, 1988; Vol. 72.

(19) Landau, L.; Lifschitz, E. Theory of Elasticity, 3rd ed.; Elsevier: New York, 1986; Vol. 7.

(20) Novoselov, K. S.; Geim, A. K.; Morozov, S. V.; Jiang, D.; Zhang, Y.; Dubonos, S. V.; Grigorieva, I. V.; Firsov, A. A. Electric Field Effect in Atomically Thin Carbon Films. Science 2004, 306, 666-669.

(21) Lefevre, R.; Goffman, M.; Derycke, V.; Miko, C.; Forró, L.; Bourgoin, J.; Hesto, P. Scaling Law in Carbon Nanotube Electromechanical Devices. Phys. Rev. Lett. 2005, 95, 185504.

(22) Wong, C. L.; Annamalai, M.; Wang, Z. Q.; Palaniapan, M. Characterization of nanomechanical graphene drum structures. $J$. Micromech. Microeng. 2010, 20, 115029.

(23) Svensson, J.; Lindahl, N.; Yun, H.; Seo, M.; Midtvedt, D.; Tarakanov, Y.; Lindvall, N.; Nerushev, O.; Kinaret, J.; Lee, S. W.; Campbell, E. E. B. Carbon Nanotube Field Effect Transistors with Suspended Graphene Gates. Nano Lett. 2011, 11, 3569-3575. 